\title{
Avaliação da força muscular respiratória em adultos cantores de coral e não cantores
}

\author{
Assessment of respiratory muscle strength in adult choir singers and non-singers \\ Evaluación de la fuerza muscular respiratoria en adultos cantantes de coro y no cantantes
}

Raysla Lorrayne Fonseca', Érica Nunes Neves², Mariana Aguiar de Matos ${ }^{3}$, Camila Danielle Cunha Neves ${ }^{4}$

\begin{abstract}
RESUMO I Um bom desempenho musical exige dos cantores alta habilidade do sistema respiratório, uma vez que, durante o canto, a musculatura respiratória produz o aumento e diminuição da caixa torácica. O objetivo deste estudo foi verificar e comparar a força muscular respiratória de indivíduos adultos cantores de coral e não cantores. Trata-se de uma pesquisa transversal, composta por indivíduos com idade entre 18 a 45 anos, de ambos os sexos, sedentários e não tabagistas, distribuídos entre dois grupos: indivíduos cantores de coral (GC; n=23) e indivíduos não cantores (GNC; n=23). Os grupos foram avaliados quanto à força muscular inspiratória (pressão inspiratória máxima - PImáx.) e expiratória (pressão expiratória máxima - PEmáx.) por meio da manovacuometria. Indivíduos do GC apresentaram valores maiores da PImáx. $(p=0,049)$ e PEmáx. $(p<0,001)$ quando comparados ao GNC. Além disso, identificou-se que os valores da PImáx. têm magnitude de efeito e poder moderados ( $d=0,56$; poder=0,59), e os valores da PEmáx. magnitude de efeito moderado e poder elevado $(d=0,77$; poder=0,82). Essa diferença das medidas de PImáx. e PEmáx. entre GNC e GC foi também observada ao se considerar os valores dos percentuais dos preditos $(p<0,05)$. A partir das análises realizadas, conclui-se que cantores adultos de coral apresentam maior força muscular inspiratória e expiratória quando comparados a não cantores.
\end{abstract}

Descritores | Pressões Respiratórias Máximas; Canto; Respiração; Fisioterapia.

ABSTRACT | A good musical performance requires singers to have high respiratory system skills since the respiratory muscles produce an increase and decrease in the rib cage during singing. Our research aimed to verify and compare the respiratory muscle strength of adult choir singers and nonsingers. This is a cross-sectional study consisting of individuals between 18 and 45 years of age, of both sexes, sedentary and non-smokers, distributed between two groups: choir singers ( $G C ; n=23$ ) and non-singers ( $G N C ; n=23$ ). Both groups were evaluated for inspiratory (maximal inspiratory pressure MIP) and expiratory (maximal expiratory pressure - MEP) muscle strength through a manovacuometer. Individuals in the GC showed higher values of MIP $(p=0.049)$ and MEP ( $p$ $<0.001$ ) when compared to the GNC. Moreover, MIP values were identified as having a moderate magnitude of effect and power ( $d=0.56$; power=0.59), whereas MEP values had a moderate magnitude of effect and high power ( $d=0.77$; power=0.82). This difference in MIP and MEP measurements between GNC and GC was also observed regarding values of the predicted percentages ( $p<0.05$ ). From the analyzes performed, one can conclude that adult choir singers have greater inspiratory and expiratory muscle strength when compared to non-singers.

Keywords | Maximum Respiratory Pressures; Singing; Breathing; Physical Therapy.

RESUMEN I Para un buen desempeño musical, los cantantes necesitan tener una alta capacidad pulmonar, ya que los músculos respiratorios durante el canto producen un aumento y disminución de la caja torácica. El objetivo de este estudio fue verificar y comparar la fuerza muscular respiratoria entre individuos adultos cantantes de coro y

'Centro Universitário Metodista Izabela Hendrix - Belo Horizonte (MG), Brasil. E-mail: raysla.fisioterapeuta@gmail.com. Orcid: 0000-0002-4580-2352

${ }^{2}$ Centro Universitário Metodista Izabela Hendrix - Belo Horizonte (MG), Brasil. e-mail: ericannunes18@gmail.com.

Orcid: 0000-0003-2599-1288

${ }^{3}$ Faculdade Sete Lagoas (FACSETE) - Sete Lagoas (MG), Brasil. E-mail: marianafisio1@yahoo.com.br. Orcid: 0000-0001-8461-5384

${ }^{4}$ Centro Universitário Metodista Izabela Hendrix - Belo Horizonte (MG), Brasil. E-mail: neves.camiladc@gmail.com. Orcid: 0000-0003-0158-7918 
no cantantes. Este es un estudio transversal, en el que participo individuos con edades comprendidas entre 18 y 45 años, de ambos los sexos, sedentarios y no fumadores, que fueron distribuidos en dos grupos: cantantes de coro (GC; n=23) y no cantantes (CNG; n=23). Los grupos se sometieron a evaluación de la fuerza muscular inspiratoria (presión inspiratoria máxima, PImáx.) y espiratoria (presión espiratoria máxima, PEmáx.) mediante manovacuometría. Los individuos del GC presentaron valores más altos de PImáx. ( $p=0,049)$ y de PEmáx. $(p<0,001)$ que los de GNC. Además, se identificó que los valores de PImáx. tienen magnitud del efecto y potencia moderados ( $d=0,56$; potencia=0,59), y los valores de PEmáx, magnitud de efecto moderado y alta potencia $(d=0,77$; potencia=0,82). Esta diferencia en las mediciones de PImáx. y PEmáx. entre GNC y GC también se observó al considerar los valores de los porcentajes de los predichos $(p<0,05)$. De los análisis realizados se concluye que los adultos cantantes de coro tienen mayor fuerza muscular inspiratoria y espiratoria que los no cantantes.

Palabras clave | Presiones Respiratorias Máximas; Canto; Respiración; Fisioterapia.

\section{INTRODUÇÃO}

O som da voz humana é gerado a partir do momento em que o ar entra nos pulmões e ao sair promove a vibração das cordas vocais, sendo uma essencial ferramenta para a comunicação humana ${ }^{1} \mathrm{~A}$ musculatura da cavidade torácica e abdominal é responsável por produzir a força necessária para direcionar o ar até as vias aéreas inferiores e, em seguida, mobilizá-lo para a laringe onde estão localizadas as cordas vocais ${ }^{2}$

Segundo Le Huche e Allali³ ${ }^{3}$ a voz pode ser considerada uma expiração sonorizada produzida pela ação dos músculos expiratórios para a expulsão do ar Durante o canto, a expiração também é um processo ativo, porém, assume uma duração maior do que na fala ${ }^{4}$. Além disso, a respiração irá variar de acordo com as exigências da apresentação musical ${ }^{4}$, envolvendo inspirações fortes e rápidas, seguida por expirações prolongadas e reguladas 5 . Nesse sentido, cada tipo de canto demanda uma forma específica de apoio respiratório, pois muda de acordo com as exigências impostas pela música para se obter a melhor emissão vocal ${ }^{1}$.

O canto coral consiste em um conjunto de cantores que realizam peças em uníssono ou em várias vozes, podendo ou não ter acompanhamento instrumental. O coral pode ser composto por vozes masculinas e femininas, os quais são agrupados conforme sua classificação vocal ${ }^{6,7}$. No canto, a função da musculatura respiratória é realizar o aumento e a diminuição da caixa torácica, sendo assim, a força da musculatura respiratória é um fator primordial no desempenho dos cantores de coral ${ }^{8}$.

A respiração adequada ao canto deve ser profunda e com o uso da musculatura inspiratória e expiratória de forma a dar apoio suficiente para a sustentação das notas $^{1,9}$. Através da consciência respiratória, adaptações e aprimoramento constante nas maneiras de exercitar o canto, como relaxamento das tensões musculares e controle respiratório apropriado, é possível observar redução da rigidez corporal e aumento da capacidade pulmonar ${ }^{10,11}$. Nesse contexto, estudos prévios relatam que cantores adultos e idosos de coral apresentam capacidade vital e inspiratória maiores quando comparadas com indivíduos não cantores, sugerindo-se assim que o hábito de cantar é provavelmente equivalente ao efeito do treinamento físico na função pulmonar, devido ao uso frequente dos músculos respiratórios ${ }^{12,13}$.

Adicionalmente, Bonilha et al..$^{5}$ demonstraram que apenas uma aula de canto foi capaz de reduzir o volume de reserva expiratório de pacientes com Doença Pulmonar Obstrutiva Crônica (DPOC), indicando assim que o canto poderia promover pequenas reduções da hiperinsuflação pulmonar dinâmica. Além disso, nesse mesmo estudo, observou-se melhora da força muscular expiratória após o treinamento de canto que, de acordo com os autores, poderia estar relacionada com o fato de a prática do canto exigir contrações sustentadas dos músculos expiratórios para gerar sons musicais estendidos.

De maneira semelhante, no estudo de Rosa et al. ${ }^{13}$ foi demonstrado o benefício do canto na força muscular expiratória de cantores idosos de coral, sendo um indício de que a atividade de canto coral pode ser benéfica para a força muscular respiratória. Assim, tem sido constatado que os volumes e capacidades pulmonares na população adulta e idosa podem ser influenciados pelo canto de coral. De modo complementar, em idosos e em pacientes com DPOC, o efeito benéfico do canto também parece estar presente na força muscular respiratória. No entanto, considerando que indivíduos idosos apresentam redução da força muscular respiratória ${ }^{8}$, os efeitos do canto nessa 
variável podem ser diferentes entre idosos e adultos. Nesse contexto, ainda são desconhecidos os impactos do canto de coral na força muscular respiratória de indivíduos adultos saudáveis. Sendo assim, este trabalho tem como objetivo verificar e comparar a força muscular respiratória de indivíduos adultos cantores e não cantores de coral.

\section{METODOLOGIA}

\section{Desenho do estudo}

Trata-se de um estudo transversal, desenvolvido no Centro Universitário Metodista Izabela Hendrix (CEUNIH), Belo Horizonte (MG). Este estudo foi conduzido de acordo com a Resolução no 466/2012 do Conselho Nacional de Saúde, Brasil. Todos os participantes assinaram o termo de consentimento livre e esclarecido previamente.

\section{Amostra}

Este estudo foi composto por uma amostra de conveniência, constituída por adultos, de ambos os sexos, alocados em dois grupos: grupo cantores $(\mathrm{GC}=23)$ e grupo não cantores $(\mathrm{GNC}=23)$. Os indivíduos cantores foram recrutados em um coral de música gospel de uma instituição religiosa e os indivíduos não cantores foram recrutados na mesma instituição religiosa e no CEUNIH. Para o recrutamento, levou-se em consideração o pareamento dos grupos com relação ao sexo, à idade e ao índice de massa corporal (IMC). O período de recrutamento da amostra e coleta de dados compreendeu de outubro a dezembro de 2019.

Os participantes atenderam aos seguintes critérios de inclusão: idade entre 18 e 45 anos; não ser fumante e não ter histórico de tabagismo; não realizar atividade física de no mínimo 30 minutos, por pelo menos três vezes semanais ${ }^{14}$; não apresentar doenças cardíacas, metabólicas ou respiratórias e não apresentar contraindicações para os testes propostos conforme publicado na literatura ${ }^{15}$. Além desses critérios, os participantes cantores deveriam comparecer regularmente nos ensaios do coral (uma vez por semana); e não exercerem outras atividades de canto ou de instrumentos de sopro. Por fim, os participantes do grupo não cantores não poderiam realizar nenhum tipo de canto. Seriam excluídos os participantes que expressassem dificuldade na compreensão e realização adequada das avaliações propostas.

\section{Procedimentos experimentais}

Todos os procedimentos experimentais foram realizados no mesmo dia. A avaliação antropométrica consistiu na mensuração do peso corporal, da estatura e do cálculo do IMC. Para medir o peso foi utilizada uma balança digital portátil (MainStays). Para avaliar a estatura foi usada uma fita métrica inelástica (Easy Read, Cateb, São Paulo/Brasil). O IMC foi determinado pelo cálculo peso corporal dividido pela estatura ao quadrado $\left(\mathrm{kg} / \mathrm{m}^{2}\right)^{16}$.

A avaliação da força muscular inspiratória e expiratória foi definida pela mensuração da pressão inspiratória máxima (PImáx.) e pressão expiratória máxima (PEmáx.), respectivamente, utilizando o aparelho manovacuômetro, modelo MRN -300/+300 $\mathrm{cmH}_{2} \mathrm{O}$ (Murenas Produtos para Saúde Ltda., Brasil). Para essa análise, os voluntários foram posicionados sentados e o nariz ocluído com um clipe nasal. A PImáx. foi mensurada a partir do volume residual e a PEmáx. a partir da capacidade pulmonar total. As manobras foram repetidas até no máximo cinco vezes, com a coleta de três manobras aceitáveis. Os esforços respiratórios máximos deveriam ser sustentados por no mínimo dois segundos, sendo consideradas aceitáveis as medidas sem vazamentos de ar e que obtiveram uma variação $\leq 10 \%$ do maior valor encontrado. Entre cada uma das medições de PImáx. e PEmáx. foi estabelecido um intervalo de um minuto para recuperação do voluntário ${ }^{15}$.

As medidas foram realizadas por examinador previamente treinado com a técnica, o qual antes da coleta ensinou e demonstrou os procedimentos a serem testados, além de ele ter utilizado comando verbal vigoroso e padronizado durante a realização do teste. Para análise dos resultados, foram considerados os valores brutos e os valores do percentual do predito, este, calculado a partir de equações específicas para a população brasileira ${ }^{15}$.

\section{Análise estatística}

Os dados foram analisados pelo programa estatístico GraphPad Prism 5.0 (GraphPad Software,Inc.,USA) e são apresentados como média \pm desvio-padrão. A normalidade dos dados foi verificada pelo teste de Shapiro-Wilk. A comparação dos resultados entre os grupos e entre os sexos foi realizada pelo teste-t independente (dados paramétricos) ou pelo teste de Mann-Whitney (dados não paramétricos).

A magnitude de efeito (d), o poder estatístico e o cálculo amostral foram examinados pelo programa 
GPower 3.1. De acordo com Cohen ${ }^{17}$, considerou-se valores de $d \leq 0,49$ como efeito pequeno; de $0,5-0,79$ como efeito moderado e $\geq 0,8$ como efeito elevado. Pelo cálculo do tamanho da amostra realizada, o qual foi baseado no estudo de Rosa et al. ${ }^{13}$, foi identificada a necessidade de no mínimo sete sujeitos por grupo. $\mathrm{E}$ foi considerado o nível de significância estatística de $p \leq 0,05$.

\section{RESULTADOS}

Foram recrutados para o estudo 51 indivíduos, sendo que desses, cinco foram excluídos por apresentarem dificuldade na realização da manobra, totalizando 23 indivíduos no GNC e 23 indivíduos no GC. Os dados demográficos e antropométricos desses indivíduos são apresentados na Tabela 1 . Observa-se que os grupos foram homogêneos em relação à idade, sexo, estatura, peso e IMC ( $p>0,05)$. O tempo médio de canto dos indivíduos foi de $9,17 \pm 5,5$ anos.

Tabela 1. Características demográficas e antropométricas dos participantes

\begin{tabular}{lccc}
\multicolumn{1}{c}{ Características } & $\begin{array}{c}\text { GNC } \\
(\mathbf{n}=\mathbf{2 3})\end{array}$ & $\begin{array}{c}\text { GC } \\
(\mathbf{n = 2 3})\end{array}$ & $\mathbf{P}$ \\
\hline Sexo $(\mathrm{M} / \mathrm{F})$ & $11 / 12$ & $11 / 12$ & --- \\
Idade (anos) & $26,00 \pm 4,00$ & $25,00 \pm 5,00$ & $0,416^{\mathrm{b}}$ \\
Estatura (m) & $1,69 \pm 0,1$ & $1,7 \pm 0,1$ & $0,524^{\mathrm{a}}$ \\
Peso $(\mathrm{kg})$ & $67,88 \pm 17,24$ & $72,17 \pm 13,48$ & $0,227^{\mathrm{a}}$ \\
IMC $\left(\mathrm{kg} / \mathrm{m}^{2}\right)$ & $23,62 \pm 4,43$ & $24,95 \pm 4,02$ & $0,292^{\mathrm{b}}$
\end{tabular}

Valores apresentados em média \pm desvio-padrão. IMC: índice de massa corporal. GNC: indivíduos não cantores de coral; GC: indivíduos cantores de coral; : teste de Mann-Whitney; ;: Test-t independente.
A Figura 1 mostra os resultados dos valores brutos da PImáx. e PEmáx. dos GNC e GC. Os indivíduos do GC apresentaram maiores valores da PImáx. $(p=0,049)$ e PEmáx. ( $p<0,001)$ quando comparados ao GNC. Além das diferenças estatísticas, identificou-se que os dados da PImáx. têm magnitude de efeito e poder moderados $(d=0,56$; poder $=0,59)$ e os dados da PEmáx. magnitude de efeito moderado e poder elevado $(d=0,77$; poder $=0,82)$.

Comportamento semelhante foi observado nas análises dos dados em percentuais dos preditos, sendo constatadas diferenças significativas nas medidas de PImáx. (GNC: $-84,31 \pm 25,96 ;$ GC: $-103,1 \pm 31,36 ; \mathrm{p}=0,032 ; d=0,67$; poder=0,72) e PEmáx. (GNC: 90,43 $\pm 26,71$; GC: 112,6 \pm $31,7 ; \mathrm{p}<0,01 ; d=0,77 ;$ poder $=0,82)$ entre os grupos. No GC, $74 \%$ dos participantes alcançaram valores superiores ao predito (>100\%) da PEmáx. e 48\% alcançaram valores superiores do predito da PImáx. Em comparação, no GNC, $30 \%$ dos participantes alcançaram valores superiores ao predito tanto da PImáx. quanto da PEmáx.

De maneira complementar, foi realizada a comparação dos resultados entre os sexos. Como esperado, em ambos os grupos os homens obtiveram pontuação mais alta que as mulheres $(\mathrm{p}<0,0001$, dados não apresentados), embora não foi observada diferença significativa no IMC (GNC, p=0,249; GC, p=0,678, dados não apresentados). Com relação às diferenças na força muscular, notou-se que apenas os valores brutos da PEmáx. do GC foram superiores nos homens em comparação às mulheres, $\mathrm{e}$ também os valores brutos de PImáx. do GNC foi superior nos homens em relação às mulheres. Não foram observadas diferenças significativas quando analisados os dados dos percentuais do predito (Tabela 2).
A

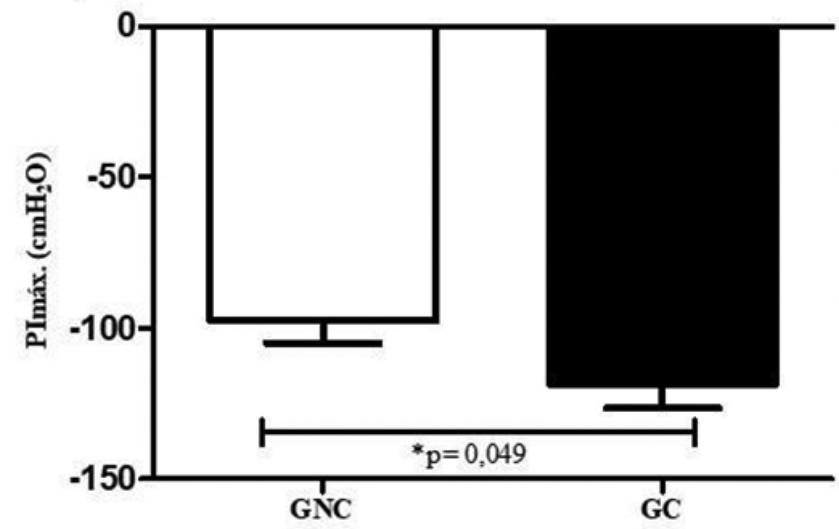

B

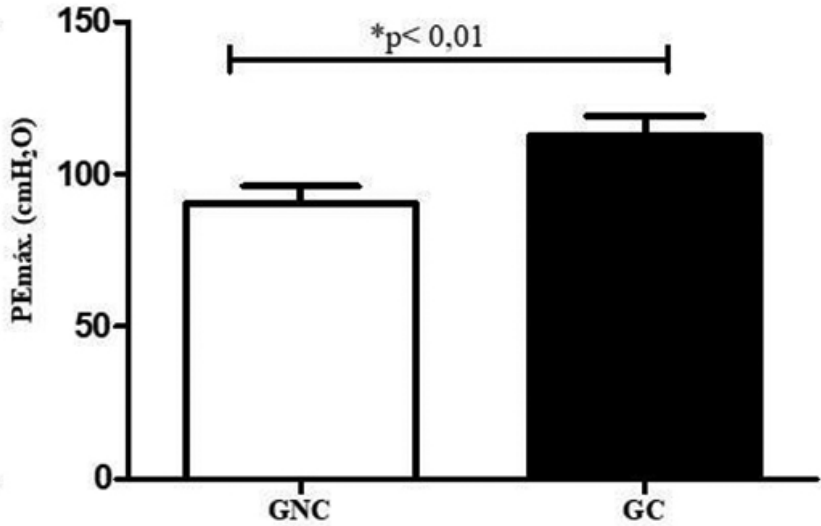

*p<0,05. GNC: indivíduos não cantores de coral. GC: indivíduos cantores de coral; PImáx.: pressão inspiratória máxima; PEmáx.: pressão expiratória máxima. Teste de Mann-Whitney.

Figura 1. Valores brutos da PImáx. (painel A) e PEmáx. (painel B) dos GNC e GC 
Tabela 2. Dados de força muscular respiratória de cantores de coral e não cantores conforme o sexo

\begin{tabular}{|c|c|c|c|c|c|c|}
\hline \multirow{2}{*}{ Variáveis } & \multicolumn{2}{|c|}{ GNC (n=23) } & \multirow{2}{*}{$\mathbf{P}$} & \multicolumn{2}{|c|}{ GC $(n=23)$} & \multirow{2}{*}{$\mathbf{P}$} \\
\hline & Masculino $(\mathrm{n}=11)$ & Feminino $(n=12)$ & & Masculino $(n=11)$ & Feminino $(n=12)$ & \\
\hline PImáx. $\left(\mathrm{cmH}_{2} \mathrm{O}\right)$ & $115,5 \pm 41,8$ & $81,11 \pm 20,28$ & $0,037^{b}$ & $130,9 \pm 46,57$ & $108,9 \pm 28,04$ & $0,301^{a}$ \\
\hline PImáx. (\% pred.) & $85,64 \pm 31,4$ & $85,61 \pm 20,10$ & $0,998^{b}$ & $96,44 \pm 34,42$ & $110,8 \pm 29,13$ & $0,334^{b}$ \\
\hline PEmáx. $\left(\mathrm{cmH}_{2} \mathrm{O}\right)$ & $96,36 \pm 28,38$ & $86,67 \pm 27,39$ & $0,450^{b}$ & $130,9 \pm 35,34$ & $100,0 \pm 13,23$ & $0,02^{\text {a }}$ \\
\hline PEmáx. (\% pred.) & $66,85 \pm 19,58$ & $87,78 \pm 29,5$ & $0,078^{b}$ & $90,28 \pm 25,89$ & $99,27 \pm 12,23$ & $0,352^{b}$ \\
\hline
\end{tabular}

Valores apresentados em média \pm desvio-padrão. GNC: indivíduos não cantores de coral; GC: indivíduos cantores de coral; a: teste de Mann-Whitney; b: Test-t independente.

\section{DISCUSSÃO}

De acordo com nosso conhecimento, este foi o primeiro estudo a investigar a força muscular respiratória de indivíduos adultos cantores e não cantores de coral. Ele demonstrou que indivíduos adultos cantores de coral apresentaram tanto força muscular inspiratória quanto força muscular expiratória superior aos indivíduos não cantores.

Existem diversos músculos cujas funções se relacionam com o processo da respiração. Os músculos intercostais externos e o diafragma são responsáveis pela inspiração, já os intercostais internos participam da expiração, que também pode ser auxiliada pela contração dos músculos abdominais (retos, transversos e oblíquos) ${ }^{18}$, levando a uma expiração forçada e mais profunda, semelhante ao que acontece durante o canto ${ }^{19}$.

Apesar de recrutar a mesma musculatura inspiratória e expiratória, falar exige basicamente $60 \%$ da capacidade vital, enquanto que para cantar a ativação dessa musculatura é em média de 70\% a 100\%, resultando em uma demanda muito maior da musculatura respiratória ${ }^{20}$. Durante o canto, a produção do som é emitida por meio da expiração ativa, porém, para que a expiração ocorra de forma eficiente e sustentada é necessária uma adequada inspiração. Sendo assim, o canto exige um apropriado suporte inspiratório e expiratório ${ }^{21}$.

Conforme os resultados deste estudo, indivíduos do grupo cantores apresentaram valores superiores de força muscular respiratória tanto na análise dos valores brutos quanto na análise dos valores do percentual do predito. Diante disso, acredita-se que a melhor força muscular inspiratória e expiratória em cantores de coral possa ser justificada pelas demandas do canto, tais como as repetidas ativações musculares, com variações da intensidade, altura, tempo de sustentação e projeção da frase musical ${ }^{1}$.

Estudos eletromiográficos sustentam essa hipótese, nos quais é reportado o aumento da atividade dos músculos respiratórios durante o canto. Neste contexto, Ramli,
Hamzaid e Engkasan ${ }^{22}$ investigaram em adultos, por meio da mecanomiografia (MMG) e eletromiografia (EMG), a atividade do esternocleidomastóideo (ECM) como músculo respiratório acessório da inspiração durante o canto. Foi observado que a atividade do ECM durante o canto foi significativamente diferente da respiração em repouso. Em outro estudo, também foi demonstrado em adultos, por meio da EMG, que na fase expiratória do canto há aumento da atividade dos músculos trapézio superior, intercostais e oblíquos laterais ${ }^{23}$.

Nossos resultados corroboram com os achados do estudo realizado por Rosa et al. ${ }^{13}$, no qual foi possível constatar que idosos saudáveis sedentários e cantores de coral obtiveram melhores resultados da força muscular expiratória quando comparados com os não cantores. Diferentemente do estudo de Rosa et al. ${ }^{13}$, o presente trabalho demonstrou que além da força muscular expiratória, coralistas adultos apresentaram força muscular inspiratória superior à dos indivíduos não cantores. Acredita-se que esses achados divergentes estejam relacionados com as diferenças de idade entre os sujeitos dos estudos e que os benefícios do canto sobre a força muscular inspiratória possam acontecer principalmente na vida adulta, momento em que a força dessa musculatura ainda está preservada.

Essa hipótese pode ser comprovada com os achados do estudo de Bonilha et al..$^{5}$ que provou o efeito do canto na força muscular respiratória de idosos com DPOC, que conhecidamente apresentam fraqueza muscular inspiratória ${ }^{24}$. Esse estudo demonstrou que idosos com DPOC que realizaram um programa de treinamento com aulas de canto apresentaram melhora da força muscular expiratória quando comparados aos pacientes controles, que realizaram apenas atividades manuais ${ }^{5}$. Assim, na população idosa, o canto parece promover aumento apenas da força muscular expiratória.

Complementarmente, neste estudo, foi observado que na análise dos dados brutos, os homens cantores revelaram força muscular expiratória superior às mulheres 
e os homens não cantores força muscular inspiratória superior às mulheres. Contudo, esse é um achado esperado, visto que conhecidamente os homens apresentam maior estatura, maior tamanho das vias aéreas, maior tamanho da caixa torácica e maior volume pulmonar ${ }^{25}$. Dessa forma, os dados do presente estudo não permitem confirmar se há diferença na força muscular entre os sexos, uma vez que os valores do percentual do predito (que leva em consideração o sexo e a idade) não foram significativamente diferentes nas medidas de força muscular inspiratória e expiratória em ambos os grupos.

Este estudo apresenta algumas limitações que devem ser pontuadas. Não foi realizada avaliação médica ou análise das capacidades respiratórias para confirmar a exclusão de desordens respiratórias, os participantes apenas declararam não apresentar e/ou não ter ciência da presença de doenças respiratórias. Além disso, não foi avaliado o nível de atividade física dos participantes por medidas diretas, tais como questionários. Apenas foi realizado o questionamento sobre o padrão de atividade física semanal e a execução de atividades esportivas, seguindo critérios que caracterizavam os indivíduos como sedentário ou fisicamente inativo. Outra questão é a ausência do cegamento do avaliador em relação aos indivíduos integrantes de cada grupo. Entretanto, para reduzir a influência do avaliador nas mensurações, este foi previamente treinado com a técnica, de forma a sempre manter o mesmo comando verbal durante a realização das medidas. Por fim, não analisamos se os músculos respiratórios do grupo de cantores apresentavam maior atividade eletromiográfica.

Destaca-se que as análises realizadas no presente estudo apresentaram poder estatístico de moderado a elevado, demonstrando os benefícios do canto na força muscular respiratória de adultos. Desse modo, acredita-se que o canto iniciado na vida adulta possa ser uma terapia alternativa para promover a manutenção ou melhora da força muscular respiratória. Para elucidar essa questão, sugere-se a realização de estudos experimentais com intuito de avaliar a força e a atividade eletromiográfica de músculos respiratórios após a implementação de um programa de canto estruturado.

\section{CONCLUSÃO}

A partir das análises realizadas no presente estudo, observou-se que adultos cantores de coral apresentaram maior força muscular respiratória, tanto inspiratória quanto expiratória, quando comparados com adultos não cantores.

\section{REFERÊNCIAS}

1. Gava W Jr, Ferreira LP, Andrada e Silva MA. Apoio respiratório na voz cantada: perspectiva de professores de canto e fonoaudiólogos. Rev CEFAC. 2010;12(4):551-62. doi: 10.1590/ S1516-18462010005000047

2. Nascimento S, Gobara ST. De onde vem o som da voz. V Encontro Nacional de Pesquisa em Ensino de Ciências - V ENPEC; 2005; Bauru, São Paulo. Atas do V ENPEC; 2006. Accompanied by: 1 CD-ROM.

3. Le Huche F, Allali A. A voz: anatomia e fisiologia dos órgãos da voz e da fala. 2. ed. Porto Alegre: Artes Médicas; 1999.

4. Branco HC. Estudo da respiração em técnica vocal [Internet]. Londrina: Universidade Estadual de Londrina; 2010 [cited 2020 Feb 20]. Available from: https://www.uel.br/pos/musica/pages/ arquivos/artigoRespiracao.pdf.

5. Bonilha AG, Onofre F, Vieira ML, Prado MYA, Martinez JAB. Effects of singing classes on pulmonary function and quality of life of COPD patients. Int J Chron Obstruct Pulmon Dis [Internet]. 2009 [cited 2020 Feb 20];4:1-8. Available from: https://www.ncbi.nlm.nih.gov/pmc/articles/PMC2672787/pdf/ copd-4-001.pdf

6. Pela SM, Behlau M, Rehder MI. O trabalho fonoaudiológico com corais. In: Marchesan IQ, Gomes ICD, Zorzi JL. Tópicos em Fonoaudiologia. São Paulo: Lovise; 1997/1998. p. 618.

7. Camargo TF, Barbosa DA, Teles LCS. Características da fonetografia em coristas de diferentes classificações vocais. Rev Soc Bras Fonoaudiol. 2007;12(1):10-7. doi: 10.1590/ S1516-80342007000100004

8. Summerhill EM, Angov N, Garber C, Mccool FD. Respiratory muscle strength in the physically active elderly. Lung. 2007;185(6):315-20. doi: 10.1007/s00408-007-9027-9

9. Fontana P, Marin L. A influência de um programa de treinamento respiratório na qualidade vocal e função pulmonar dos participantes do coral Unochapecó. FisiSenectus. 2013;1(2):2533. doi: 10.22298/rfs.2013.v1.n2.1547

10. Luiz DM, Araújo-Jorge TC, Matraca MVC. Cantando junto, por saúde e cidadania no Acre: o canto coral do IFAC em Sena Madureira. Per Musi. 2015;(32):420-38. doi: 10.1590/ permusi2015b3216

11. Degani M, Mercadante EF. Os benefícios da música e do canto na maturidade. Rev Kairós. 2011;13(2):149-66. doi: 10.23925/2176-901X.2010v13i2p\%25p

12. Irzaldy A, Wiyasihati SI, Purwanto B. Lung vital capacity of choir singers and nonsingers: a comparative study. J Voice. 2015;30(6):717-20. doi: 10.1016/j.jvoice.2015.08.008

13. Rosa CM, Celestino JPT, Colosio RC, Palácio SG. Função pulmonar e força muscular respiratória entre idosos sedentários coralistas e não-coralistas. In: V Mostra Interna de Trabalhos de Iniciação Científica [Internet]; 2010 Oct 26-29; Maringá, PR. Maringá: Cesumar; 2010 [cited 2020 Feb 20]. Available from: http://www.cesumar.br/prppge/pesquisa/mostras/ quin_mostra/juliana_policarpo_teodoro_celestino.pdf

14. American College of Sports Medicine. Diretrizes do ACSM para os testes de esforço e sua prescrição. 9. ed. Rio de Janeiro: Guanabara Koogan; 2014. 
15. Souza RB. Pressões respiratórias estáticas máximas. J Pneumol. 2002:28:S155-65.

16. Garrow JS, Webster J. Quetelet's index (W/H2): as a measure of fatness. Int J Obes [Internet]. 1985 [cited 2020 Feb 20];9(2):147-53. Available from: https://www.ncbi.nlm.nih.gov/pubmed/4030199

17. Cohen J. Statistical power analysis for the behavioral sciences. 2nd ed. Hillsdale: Lawrence Erbaum; 1988.

18. Rodrigues-Machado MG. Bases da fisioterapia respiratória: terapia intensiva e reabilitação. Rio de Janeiro: Guanabara Koogan; 2008.

19. Frigo LF. Treinamento fisioterapêutico intensivo do centro de força corporal, medidas respiratórias e vocais em cantores populares: ensaio clínico randomizado [dissertation]. Rio Grande do Sul: Universidade Federal de Santa Maria; 2016. 79 p.

20. Ray C, Trudeau MD, Mccoy S. Effects of respiratory muscle strength training in classically trained singers. J Voice. 2018;32(5):644.e25-644.e34. doi: 10.1016/j.jvoice.2017.08.005
21. Andriollo DB, Frigo LF, Moraes AB, Cielo CA. Capacidade vital forçada e pressões respiratórias máximas de cantores populares profissionais. Fisioter Bras. 2019;20(1):70-6. doi: 10.33233/ fb.v20i1.2419

22. Ramli MI, Hamzaid NA, Engkasan JP. Monitoring breathing muscle performance during singing noninvasively using mechanomyography an electromyography. J Voice. 2020;34(6):862-9. doi: 10.1016/j.jvoice.2019.06.006

23. Pettersen $\mathrm{V}$, Westgaard $\mathrm{RH}$. The association between upper trapezius activity and thorax movement in classical singing. J Voice. 2004;18(4):500-12. doi: 10.1016/j.jvoice.2003.11.001

24. Donaldson AV, Maddocks M, Martolini D, Polkey MI, Man WDC. Muscle function in COPD: a complex interplay. Int J Chron Obstruct Pulmon Dis. 2012;7:523-35. doi: 10.2147/ COPD.S28247

25. LoMauro A, Aliverti A. Sex differences in respiratory function. Breathe. 2018;14(2):131-40. doi: 10.1183/20734735.000318 